\title{
SECURE AND QOS AWARE FUZZY LOGIC BASED ROUTING FOR UNDERGROUND COAL MINE MONITORING
}

\author{
Banda Srikanth $^{1 *}$, Hemant Kumar ${ }^{2}$ and Karanam U.M Rao ${ }^{3}$ \\ ${ }^{1}$ Dept. of ECE, University College of Engineering (KU), Kothagudem, India \\ ${ }^{2}$ Dept. of Mining Engg., Indian Institute of Technology (ISM), Dhanbad, India \\ ${ }^{3}$ Dept. of Mining Engg., Indian Institute of Technology, Kharagpur, India \\ 1*bsrikanthiitkgp@kakatiya.ac.in, ${ }^{2}$ hemantismd@gmail.com, \\ ${ }^{3}$ umakaranam@gmail.com
}

\begin{abstract}
Providing safety to underground miners is a crucial and important task. Wireless Sensor Networks are widely adopted in monitoring systems due to their efficient monitoring and self-configuring nature. These networks are able to check the unattended region efficiently. In the proposed work, we deploy nodes randomly to multiple layers as tunnels and transmit sensing information from source node to destination node using WSN routing scheme. For efficient communication, we present novel routing protocol using Fuzzy Logic based systems where Fuzzy Rules are generated based on the distance from source node, energy consumption, expected energy consumption for data transmission and average energy consumption. Moreover, we present a location privacy scheme where sensitive node information and residual energy parameters are stored in hash format to maintain anonymity. The complete experimental study is simulated using MATLAB simulation tool and compared with existing protocols which shows that proposed approach achieves better performance when compared with the state-ofart techniques.
\end{abstract}

Keywords - Coal mine monitoring, wireless sensor network, energy aware secure routing, multi-layer sensor network, hybrid AES-DES algorithm and Fuzzy logic

\section{INTRODUCTION}

Coal mining system is a coherent resource for facilitating the energy/power yield, which also provides the benefit of low cost of generation and providing the significant quality fuel for several power plants. Coal mining industries are widely established in China and India. According to the study presented in [1], China produced 3,376.1 Million Metric ton coal, whereas India produced 729.8 million Metric ton and the total coal produced in 2017 is 7,548.8 Million Metric ton. These statistics show that production and demand/consumption of coal is ever increasing, due to their nature of being an efficient resource of energy. Hence coal mining industries play an important role in overall growth and economic development of the country.

On the other hand, the Bureau of Labour Statistics presented a statistical survey and concluded that underground coal mining is dangerous and hazardous task which can cause death to the miners due to the ill effects of exposure to coal dust, and

Received: March 5, 2019

Reviewed: April 21, 2019

Accepted: May 3, 2019

* Corresponding Author 
toxic gases [2]. It has also been reported that approximately $33 \%$ deaths are caused due to coal dust and toxic gases in the underground coal mines [2]. Trevits et al. [3] reported that 1601 incidents occurred in U.S alone from 1990 to 2007. Another study presented which shows that $38 \%$ percent of underground accidents occurred due to gas accumulation in the coal mines [4]. Moreover, several toxic gases also may present in the underground coal mines such as carbon dioxide $(\mathrm{CO} 2)$, carbon monoxide (CO) and hydrogen sulphide (H2S) which may cause serious effects on the human body [5]. Underground coal mining poses several other issues related to complex geological conditions which creates more hazardous environment in underground workplace. In addition to this, several natural and general aspects are also present, which can cause serious disasters in underground mines such as rock burst, water leakage, poor lighting, narrow \& complex workspace. Under such extreme condition, slight carelessness can result in serious repercussions. Thus, the accurate and prompt monitoring of underground coal mines is considered as a prime importance task for the security and safety purpose for miners.

The main aim of coal mining monitoring system is to provide desired information continuously and predict all possible disaster information such as coal dust, fire, pressure and floods etc., to warn the miners for their safety. In order to deal with these challenges, Cable Monitoring System (CMS) technology has been widely adopted for various real time underground mine monitoring systems [6]. These systems provide an efficient solution but still suffers from various complexity issues such as abrupt temperature variations, gas, pressure and variations in carbon monoxide levels. Moreover, due to the complex deployment area, several hidden challenges may also be present which may later cause serious effects. Hence, CMS technology fails to provide the solution for the complex and large tunnels. In order to deal with these limitations in underground coal mining, remote and automated systems are considered as a new solution to provide the safety and security to the miners at their underground workplace. In this perspective, wireless sensor networks (WSNs) are proven to provide promising performance in several applications such as structural health monitoring, underwater sensing and event reporting scheme because these networks can be deployed and maintained easily. Moreover, these networks are robust to hazardous incidents such as roof fall, fire, tunnel disruption, whereas wireless network technologies can be damaged due to these events. Adoption of WSN in underground mine monitoring systems is recommended recently and used for monitoring the coal mine collapses because mine collapse has highest statistics. USA and China has implemented WSN based monitoring systems in underground coal mining to minimize the deaths and injuries in the underground monitoring systems [7]. Generally, underground mines are spread in the large geographical area with multiple branches. Hence, the monitoring of this type of tunnels or mines should be robust and scalable which can help to present a robust model for underground mine monitoring system. Moreover, these mines have rough terrains and very harsh to the radio frequency based communications.

A wireless sensor network is constructed using multiple small, compact and lowpower devices which are called as sensor nodes. These sensor nodes are used for sensing the desired information, information collection, processing and transmitting to the desired destination for taking the suitable action for monitoring the environmental conditions in the deployed area [8]. In general, these networks are widely adopted in industrial, medical [9], domestic [10] and safety [11] etc. applications. In industrial applications, coal mine monitoring is considered a challenging task which needs to be addressed for minimizing the causes of underground accidents. In this work, we focus on WSN for monitoring the several parameters for underground coal mines such as gas, temperature, mine wall 
condition and fire etc. Recently, several researches have been presented in this field of coal mine monitoring using wireless sensor networks where desired data is collected, processed and transmitted to the desired location where suitable action can be taken according to the obtained information.

The complete process mainly depends on the data transmission, because during this phase several aspects need to be considered such as power consumption, network life time, node energy, data reliability and data delivery etc. In order to deal with these issue, WSN routing schemes are developed to improve the network performance in terms of network lifetime, energy consumption and packet delivery during the monitoring. However, several approaches have been presented for mine monitoring systems. But due to different challenges, such as unfavourable design of workplace, time varying channels, frequency selective channel environment and difficult terrain etc. the network maintenance issues are predominant.

\subsection{CONTRIBUTION OF THE WORK}

The main contributions of the work are as follows:

(a) Study about the underground coal mining, security aspects and techniques for underground monitoring.

(b) Development of a multi-layer sensor network model for monitoring the different levels of underground mines

(c) Development of energy efficient routing for improving the network lifetime and efficient monitoring.

(d) Better approach for cluster head selection using Fuzzy Logic by considering; energy consumption, average energy consumption, distance and expected energy consumption based fuzzy rule modelling.

(e) Implementation of SHA-256 algorithm for maintaining the location privacy along with secret data transmission using hybrid AES-DES algorithm.

\subsection{ORGANIZATION}

Rest of the article is arranged as follows: section II presents a brief discussion about recent techniques of coal mine monitoring system; section III presents proposed solution for the improving the monitoring performance, experimental study is presented in section IV and section $\mathrm{V}$ presents the concluding remark related to this work.

\section{LITERATURE REVIEW}

In this section, we briefly study about recent techniques of underground coal mine monitoring using wireless sensor networks. The complete WSN system depends on the localization and data routing schemes. Localization helps to improve the data collection and aggregation, and routing is an important paradigm to improve the network lifetime and reduces the energy consumption.

Zhao et al. [12] presented a study for underground coalmine monitoring and applications of sensor network in this field. In order to monitor the coal mine convergence, various parameters are included such as mine temperature, coal dust density, gas density and wind speed and $\mathrm{CO} 2$ density. These data are collected and transmitted towards the sink node where sensed data is fused and a final decision is acquired using Self-Organizing Map (SOM) neural network. However, due to lack of sufficient information, this system still needs some further enhancements. For monitoring the underground environmental conditions, authors developed an 
autonomous framework where mobile sensing and Ohm's law integrated model is developed for decision-making for miners.

The routing protocols developed for coal mine monitoring are mainly focused on energy balancing. Generally, the energy consumption in sensor networks are evaluated into two modes, which are active and passive mode. Passive mode energy consumption based routing schemes are widely adopted in sensor networks, where the sensor node clustering is applied to minimize the energy consumption. These protocols include LEACH [13] where cluster heads are selected periodically, PEGASIS [14] and HEED [15] etc. In addition to this, Xia et al. [16] discussed about the drawbacks of uneven clustering schemes where these clustering schemes suffer from the complexity issues which are not suitable for the underground coal mining sensor networks. In order to overcome this issue, authors have introduced UCEB-CMF protocol for improving the security and safety for coal mine monitoring systems. This approach mainly focuses on the selection of cluster head to overcome the issues of existing approaches. According to this technique, the node which is near to the sink and has highest energy is selected as cluster head. Hence, this approach presents an efficient multipath routing solution for the cluster head selection.

$\mathrm{Hu}$ et al. [17] discussed that coal mines and Goafs continuously absorb the oxygen which is used for increasing and decreasing the ignition point and increases the coal activation. At this stage, the aggregated heat needs to dissipate in a specific time-interval. If it does not dissipate properly, then it may cause coal combustion which occurs only when the temperature is higher than the critical threshold. Hence, authors focused on the development of a protocol for coal mine temperature. Moridi et al. [18] also developed an automated system for underground coal mine monitoring using WSN assisted Geographic Information System which is used for monitor and control the underground mine. In this work, authors have adopted the ZigBee networking based scheme for real-time monitoring in underground mines which are used for sensing the humidity, temperature and gas concentration and alerting for the critical conditions. This work utilizes ZigBee network approach for sensing the environmental attributes such as humidity, temperature, and gas concentration. This information is used for switching ON \& OFF fans and transmits an emergency message. In the GIS server, the monitoring plan is programmed and it can be operated if the monitored attributes are exceeding the threshold values.

Similarly, Kumari et al. [19] presented a novel approach for underground coal mine monitoring systems to provide more security to the underground monitoring system. Along with this, an authentication system was also developed. The use of WSN systems helps to achieve significant information for monitoring. In this work, authentication and network lifetime maximization are considered as a challenging issue. Hence, an authentication protocol is developed for security and light-weight and low-cost function approach is developed for reducing the energy consumption.

As discussed before, the network lifetime is also a crucial task for maintaining the efficient monitoring of underground coal mines. Several studies have been presented which shows that the network lifetime can be enhanced by using clustering schemes. Based on this assumption, Ren et al. [20] presented a novel clustering approach for a coal mine face monitoring based on the cross-layer communication strategy. Mainly this work uses management and control frames to perform the clustering. During each round of communication, cluster head $(\mathrm{CH})$ is selected and frames are utilized for estimating the channel efficiency between node and base station.

Recently, Barnwal et al. [21] studied about the issues related to underground coal mining and various advantages of WSN to monitor the coal mining to ensure the safety of miners. In this work, authors focused on the aquifer contamination 
monitoring and controlling using sensor network based schemes. WSN based system architecture is created which performs inter-cluster and intra-cluster routing scheme to improve the overall communication performance. Alfonso et al. [22] also discussed about the risks of underground coal mining and presented a monitoring strategy for monitoring the underground mines. Underground coal mining is hazardous due to different type of toxic gases which can cause serious impact on the workers. However, several approaches have been presented but implementation and operational cost becomes a challenging task. Moreover, exiting techniques only focus on the single objective; hence in this work authors presented a two-stage approach to overcome the installation and network lifetime issues. The first stage of this approach provides lowest cost solution to obtain the reduced implementation cost whereas second stage of this work helps to develop a model for network lifetime maximization using energy aware routing.

In this section, we have studied several techniques to improve the efficiency of underground coal-mine monitoring system. These techniques utilize WSN based monitoring systems where the required/desired information is sensed and transmitted to the monitoring zone using a multi-hop communication. Hence, routing of the packet plays important role, to accomplish this task. the existing techniques uses, routing schemes which are based on the artificial intelligence, linear optimization and other simplified communication approaches where achieving the desired performance in terms of energy consumption minimization is still remains a challenging task. On the other hand, security related attacks also make it more critical to implement for implementing widely in real-world scenario. Hence, there is a need to develop a robust end-to-end system which can be useful for providing efficient routing for improved and secure packet delivery.

\section{PROPOSED MODEL}

In this section, we present the proposed solution for the coal mine monitoring using WSN routing scheme. The proposed approach is carried out by developing a distributed multi-level routing for underground coal mine monitoring where multiple sink nodes are present at the different levels of the deployment. Fig. 1 shows sample network architecture with multi-level node configuration and multiple sink nodes. Here, our main aim is to develop a routing protocol which can collect the data efficiently from the Level 0 and transmit it to the multiple sinks without affecting the packet quality and a promising network lifetime of the entire network.

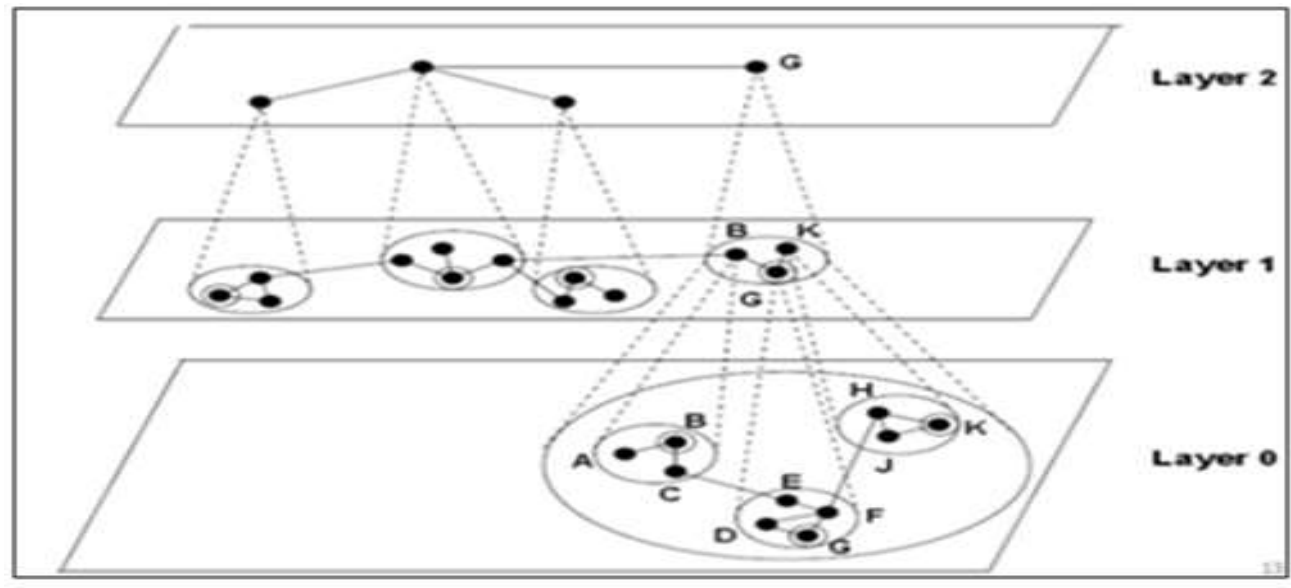

Fig. 1 Multi-Level Network Topology 
According to the proposed approach, we deploy highest possible number of sensor node at the Layer 0 because it is assumed that the Layer 0 is the region which requires maximum monitoring for safety. At Layer 0 , all the nodes collect monitoring information and the collected information is transmitted to the corresponding cluster head. Cluster head selection is considered as a tedious task in this type of scenarios due to frequent variations in the node statistics such as residual energy and sensing capacity etc. hence, here we also focus on the optimal cluster head selection which can help to improve the network lifetime.

\subsection{ASSUMPTION}

In order to achieve the desired objective, we make some assumptions for the deployed sensor network, which are as follows:

- Sensor nodes are deployed randomly along a two dimensional region.

- The overall quantity of node can be increased or decreased to make the reconfigurable WSN network architecture.

- Layer 0 contains maximum number of sensor nodes because Layer 0 is the region which requires maximum monitoring effort for safety

- The sensor nodes are heterogeneous in nature in terms of sensing capacity, energy and transmission range i.e. the nodes which are deployed at Layer 0 , require more power, sensing and transmission capacity.

- During communication phase, a routing table is managed by the cluster head where each $\mathrm{CH}$ maintains its own information, neighbouring node information, cluster head $(\mathrm{CH}) \mathrm{ID}$, neighbouring $\mathrm{CH}$ distance, Hash Function of the neighbouring $\mathrm{CH}$, and residual energy level in the cluster head.

- Each communication link is bidirectional.

\subsection{ENERGY CONSUMPTION MODEL}

During communication phase, sensor nodes require energy to perform various tasks such as data sensing, aggregation, transmitting and receiving the sensed information. We consider a radio model for presenting the energy consumption model for both free space and multi-path fading channels. The energy consumption model is computed based on the transmission of $p$ number of packets at a given distance $d$. Hence, the transmission energy can be expressed as:

$$
E_{t x}=\left\{\begin{array}{l}
\left(E_{\text {elec }}+E_{\text {amp } 1} \times d^{2}\right) \times p d<d_{0} \\
\left(E_{\text {elec }}+E_{\text {amp } 1} \times d^{4}\right) \times p d \geq d_{0}
\end{array}\right.
$$

Where $E_{t x}$ denotes the energy consumption for data transmission, $d_{0}$ denotes the threshold distance value, $E_{\text {elec }}$ denotes the required energy consumption by electronic circuits, $E_{a m p 1}$ denotes the energy required for amplification in freespace and $E_{a m p 2}$ denotes the amplification energy for multi-path model. In this model, we use the free-space model if the distance $(d)$ is smaller than the threshold distance value $\left(d_{0}=\sqrt{\frac{\epsilon_{f s}}{\epsilon_{m p}}}=\sqrt{\frac{10 * 10^{-12}}{0.0013 * 10^{-12}}}=87.70\right)$ otherwise we apply multipath modeling for energy consumption estimation. Similarly, the total energy consumed during receiving the data can be given as: 


$$
E_{r x}=E_{\text {elec }} \times p
$$

\subsection{ENERGY CONSUMPTION PREDICTION}

In this section, we present an energy consumption prediction model for the nodes which are present in the layer. Before cluster formation, total quantities of members in the cluster are unknown. However, it can be estimated based on the neighbours of the potential $\mathrm{CH}$. In this work we present a model to predict energy consumption during communication phase. However, this process is implemented, once the cluster formation the data transmission phase is divided into multiple frames where sensor nodes transmit the data to the corresponding cluster head during their assigned transmission time slot. Let us consider that in a given time slot $t$, total $n$ number of cluster members are present along with their cluster head. During communication, data is transmitted to the corresponding cluster head in the assigned time slot duration. Let us consider that a $\mathrm{CH}$ has $n$ number of clusters memebrs in a given time frame and receives $n$ number of messages from these cluster members. The received messages are transmitted to a destination node or base station, located at distance $d$. Based on this, the total number of frames can be computed as:

$$
\text { Num }_{\text {Frame }}=\frac{t_{\text {ss }}}{t_{\text {slot }} * n+t_{\text {CHtoBS }}}
$$

Where $t_{s s}$ denotes the steady state phase operation time, $t_{\text {slot }}$ denotes the slotted time requirement for transmitting the data from cluster members to $\mathrm{CH}$ and similarly, $t_{C H \text { toBS }}$ denotes the time required for transmitting the data from $\mathrm{CH}$ to base station. Based on these assumptions, the expected energy consumption can be computed as:

$$
\left.E_{\text {exp }}\left(b, d_{B S}, n\right)=\text { Num }_{\text {frame }} *\left(E_{T x}\left(b, d_{B S}\right)+E_{R x}(l) * n\right)\right)
$$

Where $b$ denotes the size of data which is similar for all during transmission and receiving the data, distance to base station can be computed based on the coordinate systems. With the help of these parameters, the expected energy consumption can be expressed as:

$$
E_{\text {exp_res }}\left(b, d_{B S}, n\right)=E_{\text {residual }}-E_{\text {exp }}
$$

Where $E_{\text {residual }}$ denotes the remaining energy level of the node.

\subsection{MULTILAYER CLUSTER FORMATION AND HEAD SELECTION}

In this work, we consider a multilevel network deployment for underground coal mining where multiple nodes are present at each level of the network. Hence, our first aim is to identify the location of node position as source node and its relative layer. Let us consider that the network is divided into $L$ number of layers with an interference range of $d_{0}$, and $d(s, i)$ denotes the distance between source node $i$ and sink node $s$. The node belongs to the layer $L_{i}$ can be expressed as:

$$
L_{i}=\left[2 * \frac{d(s, i)}{d_{0}}\right]
$$

Where $d_{0}$ denotes the interference range and $L=\max \left(L_{i}\right)$ where $i=1,2, \ldots$ However, we have assumed that multiple number of nodes can be present in the Layer 0 , where multiple cluster heads also can be present due to different energy capacity and transmission range, hence, we compute the maximum possible number 
of cluster heads in the layer $L_{i}$. The total number of cluster head in the layer depends on the number of nodes in the layer and distance between layer and sink node. The total number of possible cluster heads in the layer $L_{i}$ can be computed as:

$$
C H(i)=\left[\alpha * \frac{N_{i}}{L_{i}}\right]
$$

Where $\alpha$ is an adjustable parameter whose value is between 0 to 1 . In our work, we considered $0.5,0.7$ and 0.9 for identifying the optimal number of cluster heads. In order to form the cluster, we consider a range based approach where a range is fixed for each layer and the nodes which are in this range are called cluster members. At this stage, we have obtained following parameters: network information i.e. deployment related information, number of layers, the corresponding node to the layers, distance between nodes, energy consumption modelling, expected energy consumption and possible number of cluster heads in the current layer.

Based on these parameters, we present a Fuzzy logic based approach for cluster head selection. Fuzzy Logic model is known as a non-linear mapping data mapping techniques where input data is provided in the form of vector and it provides the output in scalar form where the inputs and outputs are in the form of linguistic variables. According to this process, fuzzy rules need to be defined and the complexity of these rules depends on the input parameters and their fuzzy sets.

After formulating the fuzzy rules, fuzzy inference system combines these rules and generates a single fuzzy set for achieving the output. Generally, this process is implemented for the application scenarios where more uncertainties and imprecise information is present. It can be applied for various domains such as medical systems, pattern recognition, robotics and optimization etc. This system architecture contains three main components as depicted in Fig. 2.

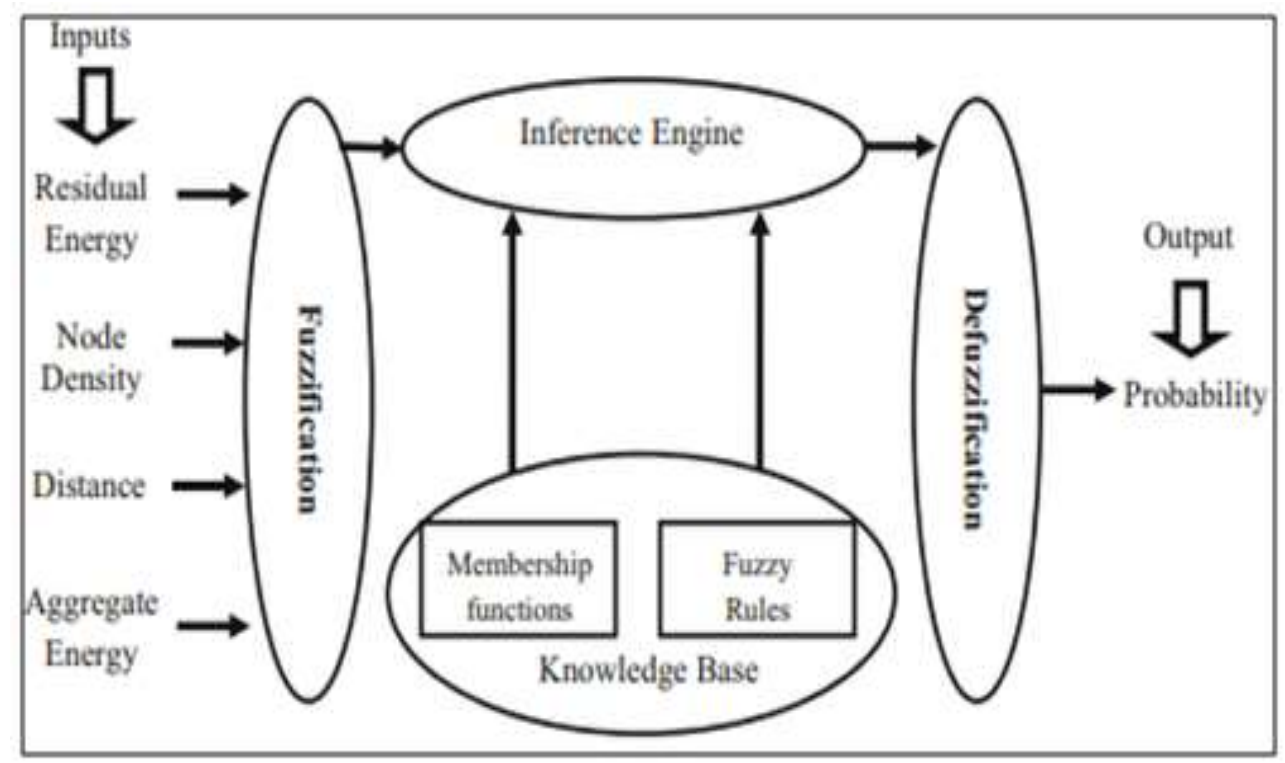

Fig. 2 Fuzzy Logic Architecture

The main components of fuzzy logic are termed as fuzzifier, which helps to convert the crisp value into membership degree by using the corresponding membership function. The membership functions are used for determining the relationship between crisp value and its corresponding specific linguistic value. 
Inference system: This component is used for making critical decisions, based on which the formulation is carried out using different linguistic variables and these linguistic statements are stored in a rule base. These fuzzy rules are formulated in the form as "Premise: $\mathrm{x}$ is A., Implication: IF $\mathrm{x}$ is A THEN $\mathrm{y}$ is $\mathrm{B}$ and Consequent: $\mathrm{y}$ is $\mathrm{B}$ ", these rules are composed of input variables which are connected using logical function (AND, OR, NOT) and the Consequent is an output variable. Let us consider that a qinput 1-output Fuzzy Logic system is given as

R: Var 1 is Member $_{1}^{i}$ and $\operatorname{Var}_{2}$ is Member $_{3}^{i}$ and $\ldots \operatorname{Var}_{q}$ is $\operatorname{Member}_{q}^{i}$ then output is $O^{\prime}$. Using Fuzzy logic, for inputVar' $=\left\{\operatorname{var}_{1}^{\prime}, \operatorname{var}_{2}^{\prime}, \operatorname{var}_{3}^{\prime}, \ldots \operatorname{var}_{q}^{\prime}\right\}$, the degree of fuzzy rules provides the output as:

$$
\begin{gathered}
\psi_{\text {Memberi }_{1}^{i}}\left(\operatorname{var}_{1}^{\prime}\right) * \psi_{\text {Member }_{2}^{i}}\left(\operatorname{var}_{2}^{\prime}\right) * \psi_{\text {Member }_{3}^{i}}\left(\operatorname{var}_{3}^{\prime}\right) * \ldots \\
* \psi_{\text {Member }_{q}^{i}}\left(\operatorname{var}_{q}^{\prime}\right)=T_{l}^{q} \psi_{\text {Member }_{1}^{i}}\left(\operatorname{var}_{1}^{\prime}\right)
\end{gathered}
$$

Where $\psi$ denotes the membership function, $*$ denotes triangular membership function and $T$ denotes the trapezoidal membership norms which are the binary operations applied to the fuzzy sets for membership function.

Defuzzification: This is a process which is used for obtaining the crisp values from fuzzy sets. Several methods are present for defuzzification of the fuzzy sets such as centre of gravity, maximum method and centre of singleton. These methods are used for identifying the output based on the maximum activity of fuzzy sets and it provides the probability of output by computing the centroid which can be given as:

$$
\text { Centroid }=\frac{\sum \psi_{n}(a) * a}{\sum \psi_{n}(a)}
$$

In this work, we utilize Fuzzy Logic system for cluster head selection where multiple input parameters are considered to select the cluster head, these parameters are: (a) distance to the sink node, (b) residual energy (c) expected energy consumption. Based on these parameters, we designed the fuzzy sets for each parameter by using different membership functions. Fig. 3 shows a Fuzzy logic based architecture for cluster head selection using aforementioned Fuzzy inputs.

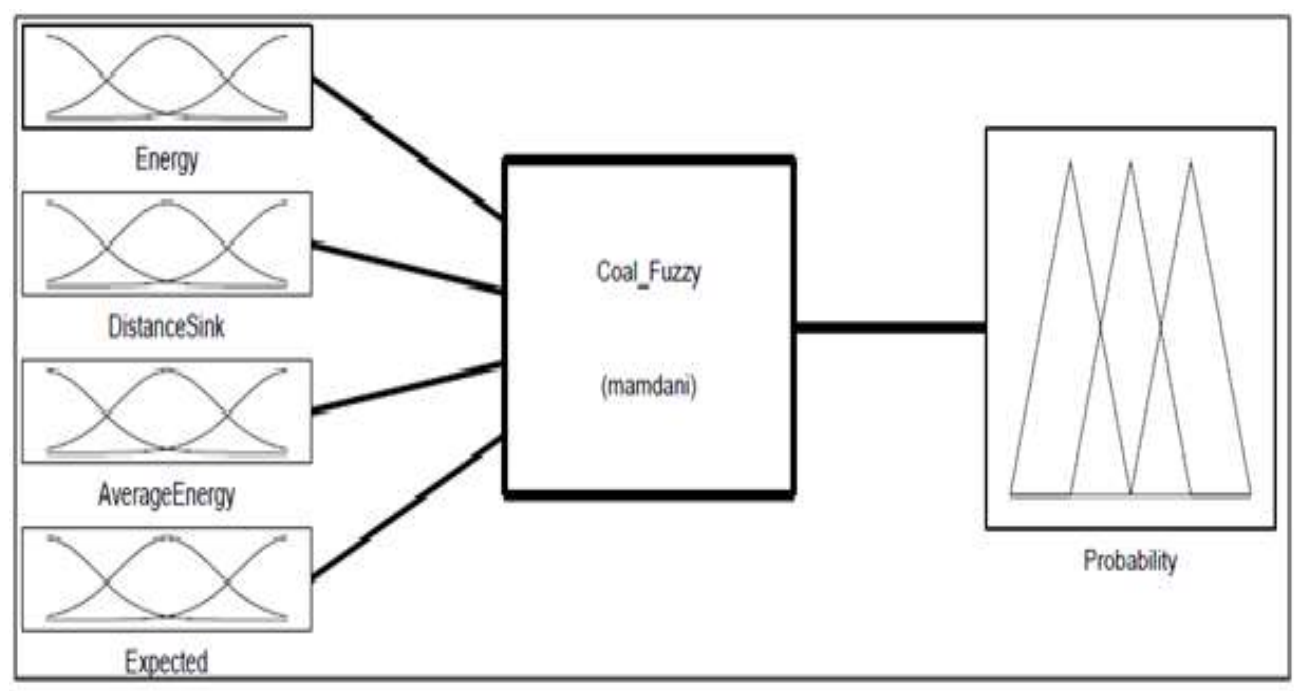

Fig. 3 Coal Mine Monitoring Fuzzy Logic Architecture 
First input to the FL system is considered as Residual energy in the cluster head which is mainly divided into three levels as "Low", "Medium" and "High". In this input, "Low" and "High" input considers the trapezoidal membership function and "Medium" level uses triangular membership function for computing the centroid based on the energy. Fig. 4 represents the membership function representation of "Energy" input.

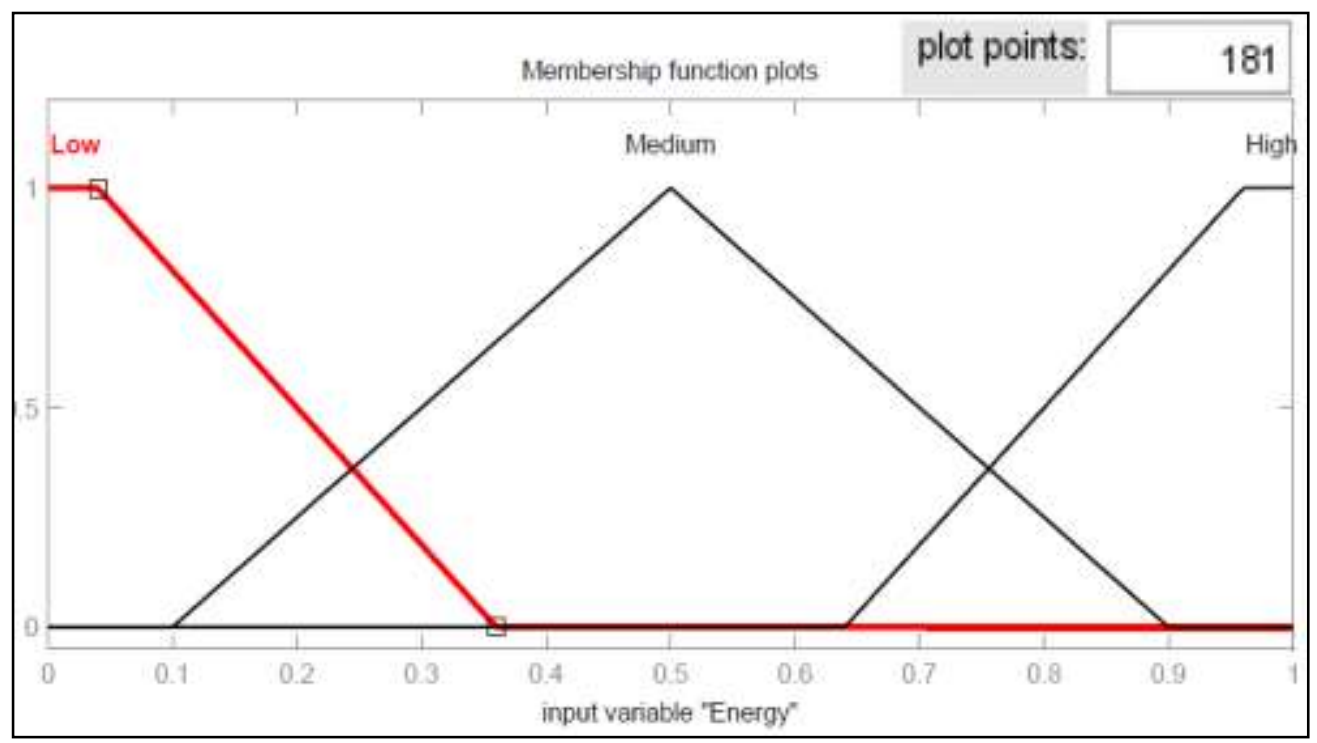

Fig. 4 Residual energy Membership function

Distance from the sink node is an important parameter which is used for cluster head selection. While moving from source node to destination node, we follow multi-hop path passing through the cluster heads. At this stage, the cluster head which is having less distance from the sink node has the higher probability to be selected as cluster head. The membership function representation is depicted in Fig. 5 .

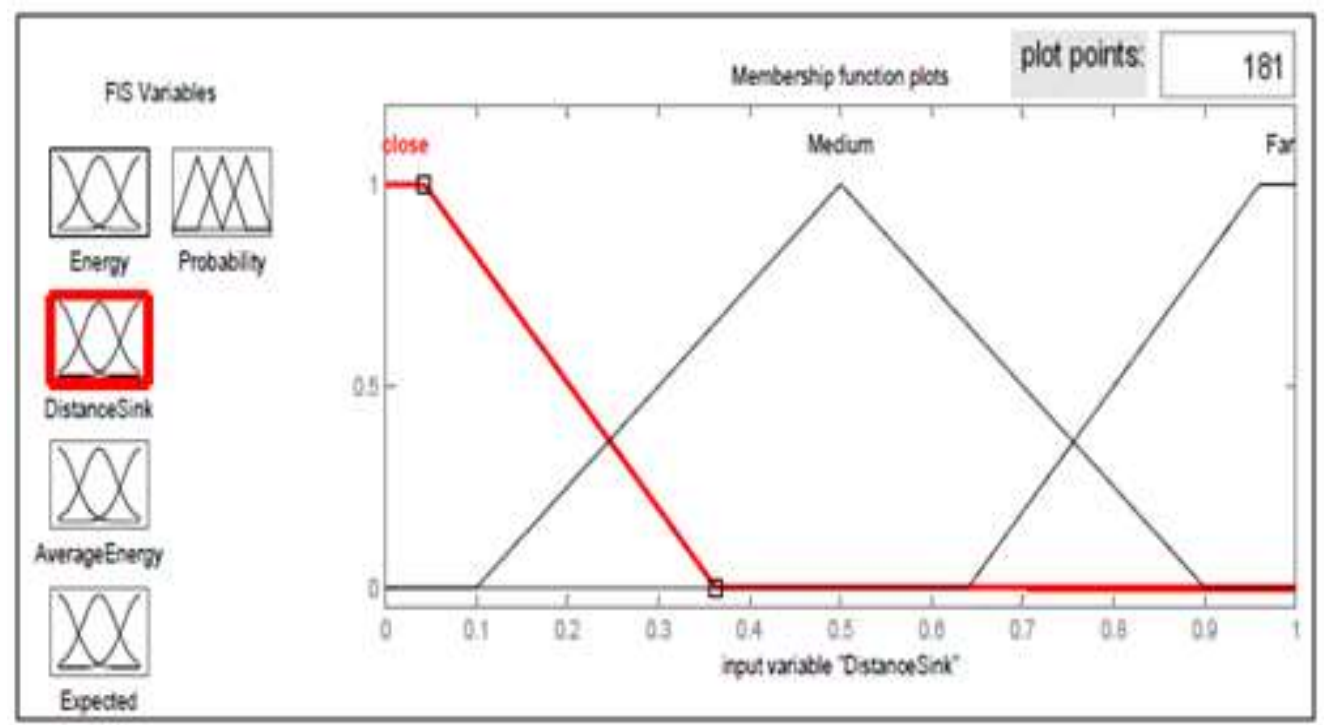

Fig. 5 Distance from the sink node membership function. 
In this membership, three stages of distance are considered as close, which shows that current $\mathrm{CH}$ is closer to the sink node, medium which implies that the distance $\mathrm{CH}$ is located at a medium distance according to its transmission range and far membership function which shows that the $\mathrm{CH}$ is located far and also it is not present in the communication range. In this also, "close" and "Far" membership function uses trapezoidal function whereas "medium" distance function uses triangular membership function.

The next parameter is considered as average energy consumption during communication including data sensing, processing and transmitting to the next hop. Fig. 6 represents the membership function based probability for cluster head selection based on the three level of energy which are called as "Less", "Medium" and "Elevate".

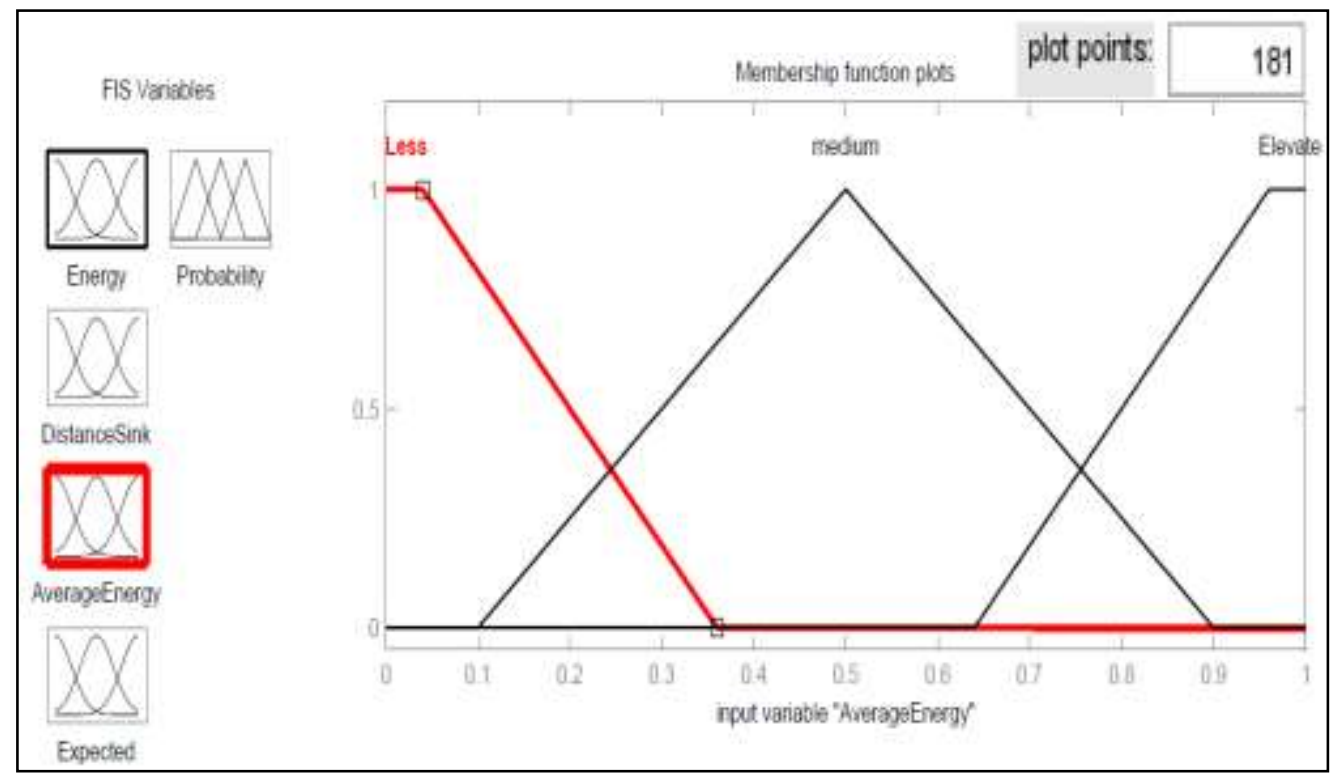

Fig. 6 Average energy level membership function

In this membership "Less" and "Elevate" membership functions use trapezoidal membership function whereas "medium" distance consuming membership function uses triangular membership function. Hence, the cluster which is having higher energy has more probability to become the next hop and cluster head can be selected from that cluster based on its energy parameters.

Similarly, we present the Fuzzy logic based membership function representation as depicted in Fig. 7. This contains three levels for rule formation which are called as "Less", "Average" and "More". In this also, low and high points use trapezoidal membership function where as "Average" energy consumption uses triangular membership function. According to this rule, the $\mathrm{CH}$ which has higher energy consumption expectation is not selected for the $\mathrm{CH}$. 


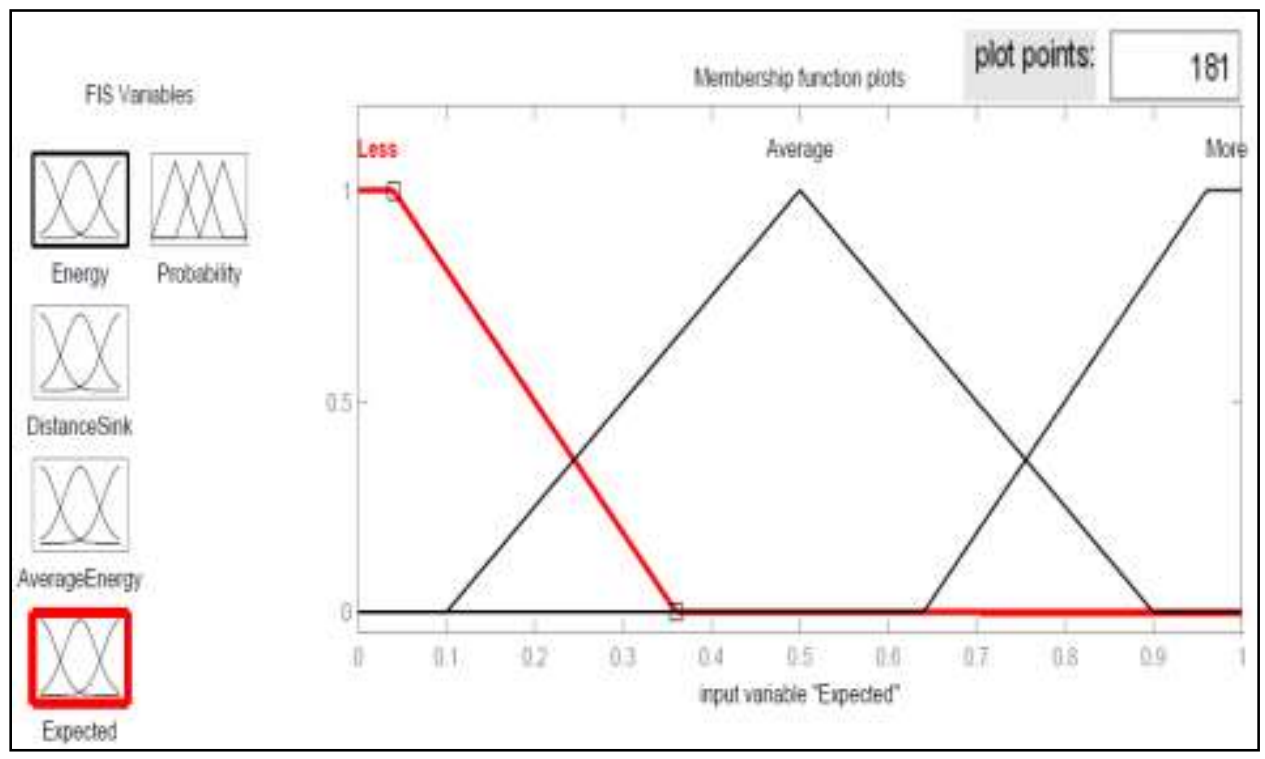

Fig. 7 Expected energy consumption membership function

Let us consider that total $N$ numbers of nodes are present, where $c$ nodes are assigned as cluster heads. During communication phase, cluster members dissipate the energy for data sensing, information collection and send the aggregated information to the cluster head. Later, cluster heads consume some amount of energy for aggregation of the data and transmitting the data to the sink node. In the first round or iteration of communication, data collection by sensor nodes, transmitting the data to the corresponding cluster head, data aggregation by cluster head, transmitting the data from $\mathrm{CH}$ to the sink node using multihop routing, tasks are performed and the data transmitted is called in one iteration is known as one frame. Let us consider that a cluster member collects the $B$ bits of data in each iteration, the energy consumption for this can be expressed as:

$$
E_{c m}=B\left(\epsilon_{\text {elec }}+\left(\epsilon_{f s} * d^{2}\right)\right)
$$

Similarly, cluster head consumes energy for data aggregation and transmission which can be computed as:

$E_{c h}=B * \epsilon_{D A} *\left(\frac{N}{c}-1\right)+B * \epsilon_{\text {elec }}+B * \epsilon_{m p} * d^{4}+B * \epsilon_{\text {elec }}\left(\frac{N}{c}-1\right)$

Based on (11), the energy consumption for receiving the data from sensor nodes is computed as $B * \epsilon_{\text {elec }} *\left(\frac{N}{c}-1\right)$, energy consumption for data aggregation is given as $B * \epsilon_{D A} *\left(\frac{N}{c}-1\right)$ and energy consumed for data transmission is computed using $\left(B * \epsilon_{\text {elec }}+B * \epsilon_{m p} * d^{4}\right)$

Probability $=\frac{L_{r e} * a+L_{e x p} * b+L_{a e} * c+\left(M_{d}-L_{d}\right) * d}{M_{r e} * a+M_{\text {exp }} * b+M_{a e} * c+M_{d} * d}$

Where $L_{r e}$ denotes the level of residual energy, $L_{\text {exp }}$ denotes the level of expected energy consumption, $L_{a e}$ denotes the level of average energy consumption, $M_{\text {exp }}, M_{a e}$ and $M_{r e}$ denotes the maximum expected energy consumption value, maximum average energy and maximum residual energy. 
Based on the maximum probability, the cluster head can be selected and data can be transmitted from one $\mathrm{CH}$ to next $\mathrm{CH}$.

\subsection{PACKET ROUTING SCHEME}

Based on these assumptions, we implement a path discovery phase where a set of neighbouring node is created which forwards the data from source to sink node. The paths are constructed in multi-path and node-disjoint which shows that nodes don't have a common source and destination. In this work, node-disjoint path communication is preferred because it can help to improve the bandwidth utilization and are fault-tolerant in nature. Moreover, if one path or node fails, that particular path only will be affected and communication quality will not degrade due to multipath transmission. In this process, first of all, the process is initiated where each node transmits a HELLO message to obtain the network information and identify the neighbours which can be used for communication. Each communicating node is responsible to maintain and update neighbouring node table which contains the complete information about the neighbouring sensor nodes. Fig. 8 shows a general structure of HELLO message.

\begin{tabular}{|l|l|l|l|}
\hline Source ID & Destination ID & Route ID & Residual Energy \\
\hline
\end{tabular}

Fig. 8 HELLO message structure

Communication link quality is evaluated in terms of SNR (Signal-to-Noise Ratio) for the link between node and its neighbouring node and hop counts provides the distance information in terms of hops from its originator. In the next phase, we focus on the primary path computation phase, where the sink node computes the most preferred next hop with the help of link cost function by transmitting RREQ message to the most suitable hop. Fig. 9 shows structure of RREQ message.

\begin{tabular}{|l|l|l|l|l|l|l|}
\hline Source ID & Destination ID & Route ID & Residual Energy & Expected Energy & Average energy & Distance \\
\hline
\end{tabular}

Fig. 9 RREQ message architecture

This process is repeated until the complete path is identified. Later, we perform selection of alternate path where a separate RREQ message is transmitted to find the alternate path from the neighbouring nodes. In order to avoid the path sharing, RREQ message acceptance is limited to 1 for each node. A sample example of path discovery is depicted in Fig. 10.

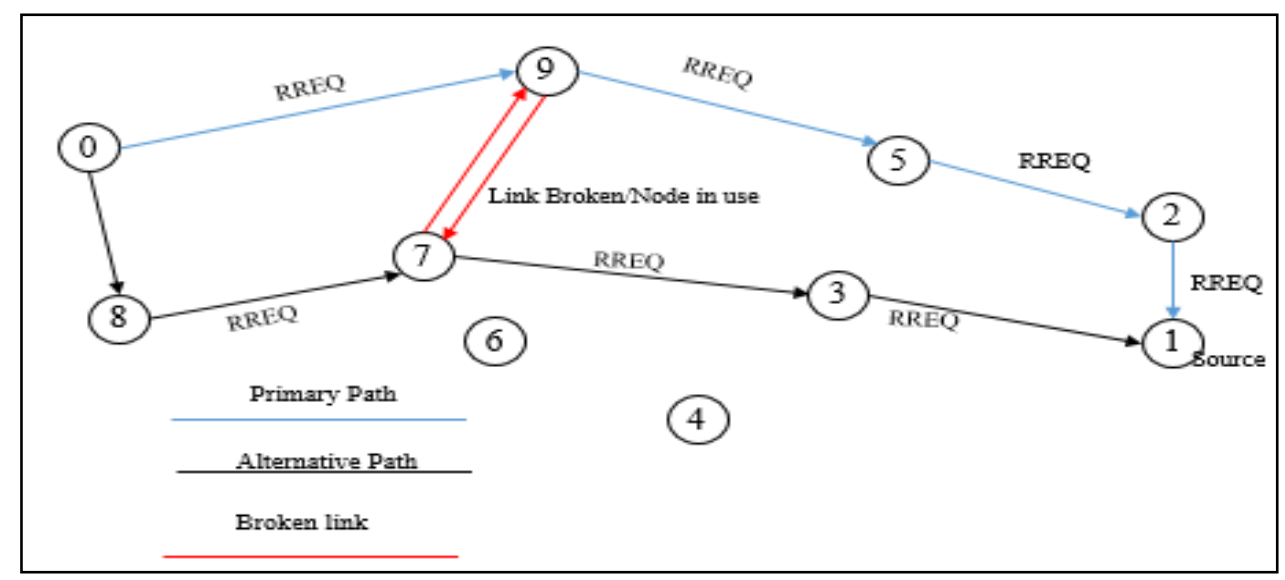

Fig. 10 Path discovery 
According to the path discovery example, sensor node 9 finds the next preferred node as node 7 and sends a RREQ message but node 7 is appended into the main path hence it responds with a busy response. Later, node 9 selects the next preferred node with the help of RREQ message and continues the communication.

Finally, the path selection phase is carried out to obtain the information about total number of required path for transmitting the packets from source to the destination. In order to find the total number of required path, a probability $p_{i}$ is described which provides the information about successful message delivery. With the help of this, the total number of required paths can be computed as:

$$
k=x_{a} \cdot \sqrt{\sum_{i=1}^{N} p_{i}+\sum_{i=1}^{N} p_{i}\left(1-p_{i}\right)}
$$

Where $x_{a}$ denotes the normal distribution bound limits. From (15) we obtain total $k$ number of paths in which $l$ number of paths are selected for real -time and nonreal-time traffic transmission. The total paths can be expressed as:

$$
l=\frac{T}{T_{n r}+T_{r}}
$$

Where $T_{n r}$ denotes the non-real-time traffic and $T_{r}$ denotes the real-time traffic.

In this work, we focus on the location privacy also to maintain the security. Hence, each node ID, destination ID and route ID is assigned with a Hash function and other information available in routing table are encrypted using hybrid AES DES algorithm.

\section{RESULTS AND DISCUSSION}

In this section, we discuss about the experimental study and the performance of proposed approach for underground coal mine monitoring application. The complete experimental study is carried using MATLAB simulation tool installed on the Windows platform. Various simulation parameters are considered in this study to measure the performance of proposed model as presented in Table I.

Table I. Simulation Parameters

\begin{tabular}{|l|l|}
\hline Network area & $1500 \times 1500 \mathrm{~m}^{2}$ \\
\hline Number of nodes & 100 \\
\hline Number of sinks & 1 \\
\hline Transmission range & $25 \mathrm{~m}$ \\
\hline Packet Size & 1024 bytes \\
\hline Transmit Power & $15 \mathrm{~mW}$ \\
\hline Received Power & $13 \mathrm{~mW}$ \\
\hline Ideal Power & $12 \mathrm{~mW}$ \\
\hline Sleep mode Power & $0.015 \mathrm{~mW}$ \\
\hline Initial Battery power & 100 joules \\
\hline Max Buffer Size & $256 \mathrm{~KB}$ \\
\hline
\end{tabular}

The performance of proposed approach is carried out in terms of network lifetime, average number of packets delivered to base station, packet drop, and endto-end throughput of the entire network. 


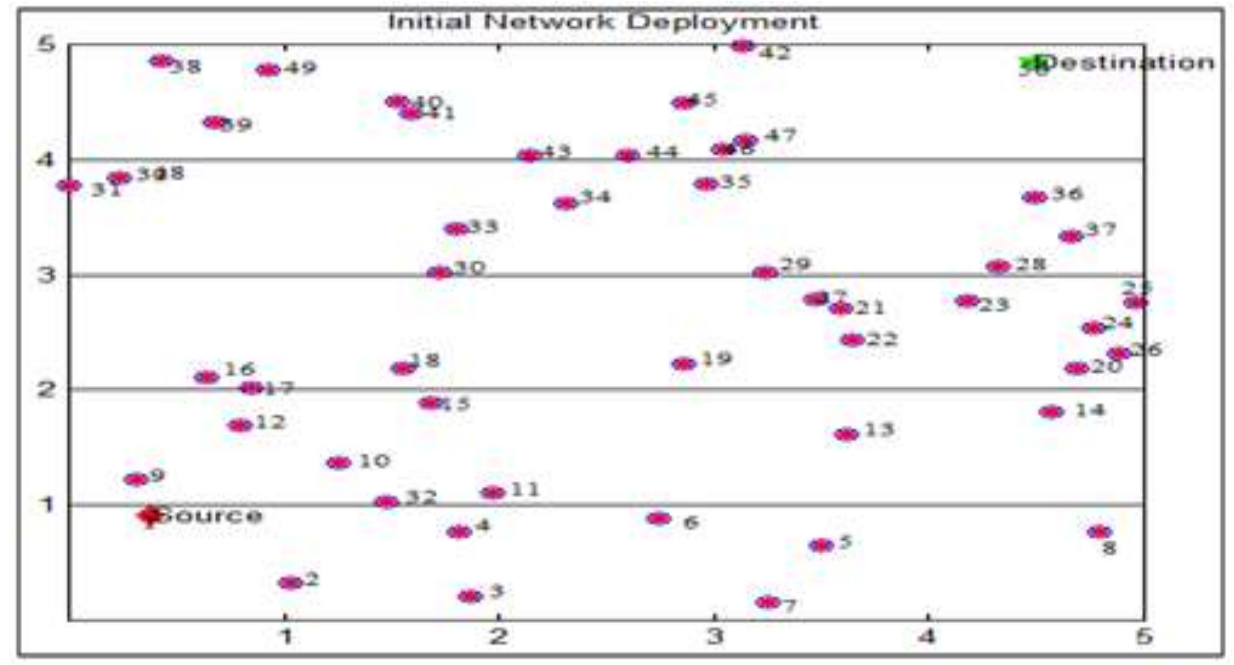

Fig. 11 Initial Network Deployments

Fig. 11 shows initial network deployment with 5 layers, where source node or Initial cluster head is deployed in the layer 0 , which is further selected as the source node and the destination or sink node is at layer 4 . In the layer 0 , total 8 numbers of nodes are present. The corresponding energy level and their Hash IDs are presented in Table II.

Table II. Layer 0 Node Information

\begin{tabular}{|c|c|c|}
\hline Node ID & Hash ID & Energy Level \\
\hline 1 & dc1c320defa535b9d8177fc07ace6481 & 95 \\
\hline 2 & a8cf591c39650d4614dd461b48dca374 & 85 \\
\hline 3 & f9cda26033ff0ad05e0884f4ca466113 & 82 \\
\hline 4 & 183d1d6239db5dde488f74bdf0619ff1 & 86 \\
\hline 5 & 6bc3b2744d8299ec3a3d1e229465429d & 81 \\
\hline 6 & 0aff29077259eba14cb1e657a4fc4a34 & 85 \\
\hline 7 & e65fffdfdaf7715b168c35a564e28418 & 86 \\
\hline 8 & dcd75cedc265cd8c7bce6aae20dfbcf7 & \\
\hline
\end{tabular}

\subsection{LAYER 0 NODE INFORMATION}

According to the Table II, node 1 has the maximum energy level hence it is selected as cluster head in the layer 0 of the network.

\subsection{LAYER 1 NODE INFORMATION}

In this layer also, 8 number of node are present whose corresponding Hash ID and energy levels are presented in Table III.

Table III. Layer 1 Node Information

\begin{tabular}{|c|c|c|}
\hline Node ID & Hash ID & Energy Level \\
\hline 9 & aa954aa28e1148b7bde114072cfb4cec & 86 \\
\hline 10 & 7fa635a1652b1a3f13a38fae00876101 & 87 \\
\hline 11 & e2b0e799db1b8bd698510321a8bf0961 & 82 \\
\hline 12 & f55f6a8b97afba0c508ec4ff46be44c9 & 84 \\
\hline 13 & 7ae5d7213d81950a64fcb88fc5847fd5 & 88 \\
\hline 14 & 050974675573a824daecd8df177d9812 & 83 \\
\hline 15 & d86496b6f9bc3df113bffbbd300b0fc8 & 84 \\
\hline 32 & 62eaf2b191e4d1fb262a47f7bbc74dab & 90 \\
\hline
\end{tabular}


According to the configuration presented in layer 1, Node 32 has the highest energy level. Hence, it is selected as cluster head. Similarly, this process is repeated until the destination node is obtained. Fig. 12 shows the complete routing model using proposed approach.

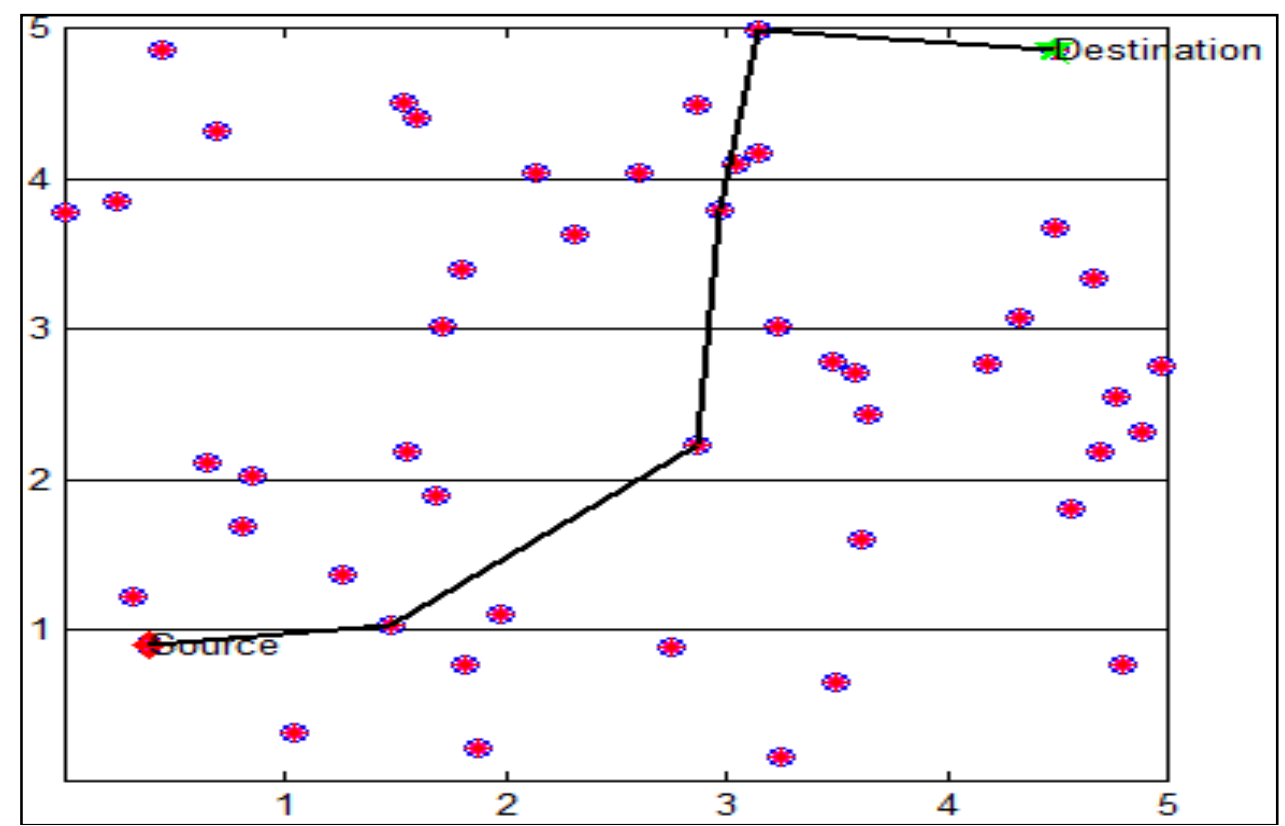

Fig. 12 Proposed routing scheme

In this work, we use fuzzy logic system for cluster head selection. Total 5 clusters have been selected based on the membership functions which are presented in Table IV.

Table IV. Fuzzy Logic based decision table for the cluster head selection

\begin{tabular}{|c|c|c|c|}
\hline $\begin{array}{c}\text { Cluster Head } \\
\text { Number }\end{array}$ & C1 & C2 & C3 \\
\hline CH1 & H & M & M \\
\hline CH2 & H & H & M \\
\hline CH3 & L & H & M \\
\hline CH4 & L & L & L \\
\hline CH5 & H & M & L \\
\hline CH6 & H & L & H \\
\hline CH7 & H & L & L \\
\hline CH8 & H & L & H \\
\hline CH9 & H & L & M \\
\hline CH10 & H & & \\
\hline
\end{tabular}

\subsection{PERFORMANCE MEASUREMENT}

First of all, evaluate the performance in terms of network lifetime for varied number of nodes using proposed approach. Performance of proposed approach is compared with the existing routing protocols such as LEACH (Low-energy adaptive clustering hierarchy), M-LEACH (Modified Low-energy adaptive clustering hierarchy), DEEC (distributed energy-efficient clustering algorithm) and SEP (Stable Election Protocol) protocols. 


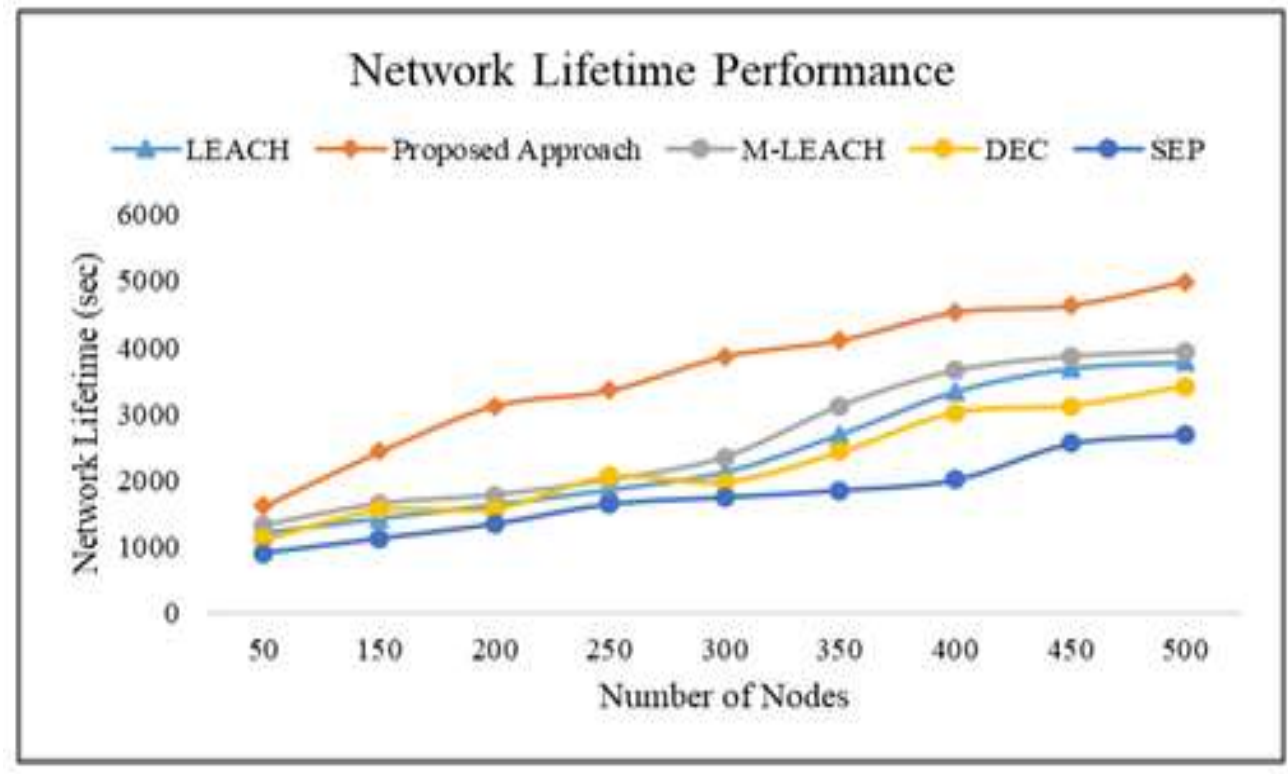

Fig. 13 Network lifetime performance

The Fig. 13 shows a comparative performance in terms of network lifetime. The comparative study is carried out for varied number of nodes. The performance obtained using proposed approach shows improved results when compare to existing protocols as mentioned before. The average network lifetime using LEACH is 2413.6, M-LEACH is 2632, DEC is 2255 , SEP is 1768 and proposed approach is 3622 which shows that proposed approach achieves better performance when compared with the other routing protocols.

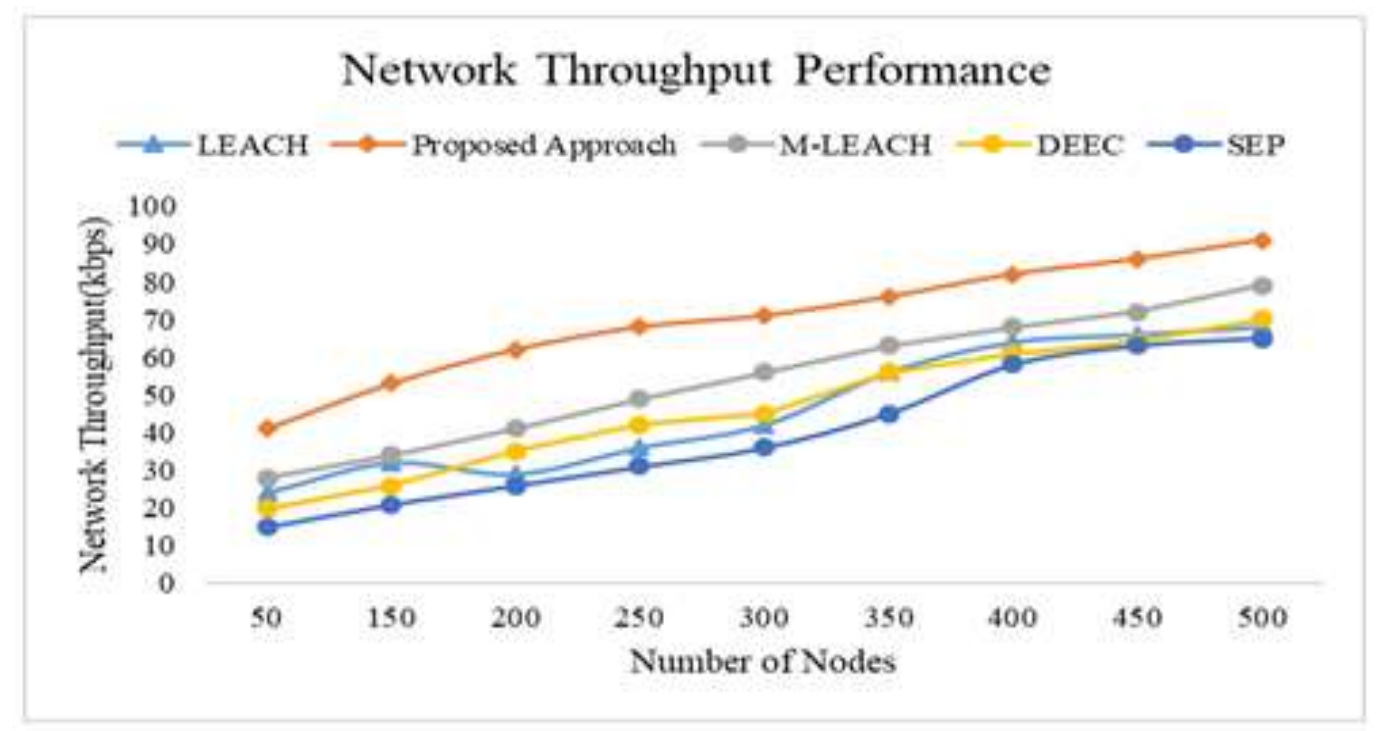

Fig. 14 Network Throughput

Fig. 14 shows network throughput performance for varied number of nodes. As the numbers of nodes are increasing, the overall throughput of the network increases due to decrement in the node death rate hence network lifetime is prolonged and the overall throughput on the network also increases. The average 
throughput is obtained as 40, 46.3, 54.4, 46.5 and 70 using SEP, LEACH, MLEACH, DEEC, and Proposed Approach.

During communication phase, the overall energy consumption and residual energy is also evaluated and compared with the existing LECH protocol. Fig. 15 shows a comparative performance in terms of average energy consumption for 1000 round scenario in for varied number of nodes. The average energy consumption performance is obtained as 338.77, 312.56, 266.5, 229.2 and 186 using SEP, DEEC, LEACH, M-LEACH and Proposed Approach.

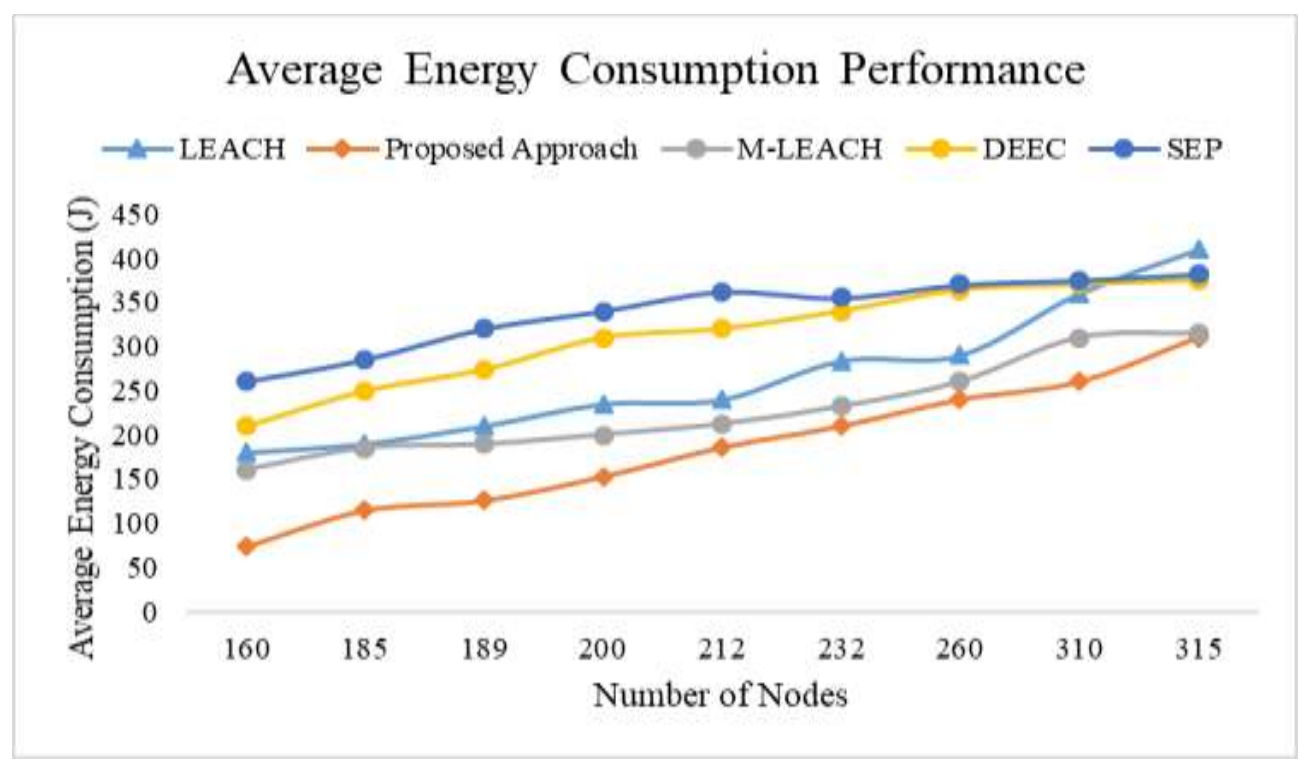

Fig. 15 Average energy consumption performance

This comparative study shows that the proposed approach reduces average energy consumption when compared with the existing LEACH protocol. Similarly, Fig. 16 shows a comparative performance in terms of average residual energy where proposed approach achieves improved performance and it can be concluded that energy wastage is less in proposed approach. This helps to improve the network lifetime and network throughput.

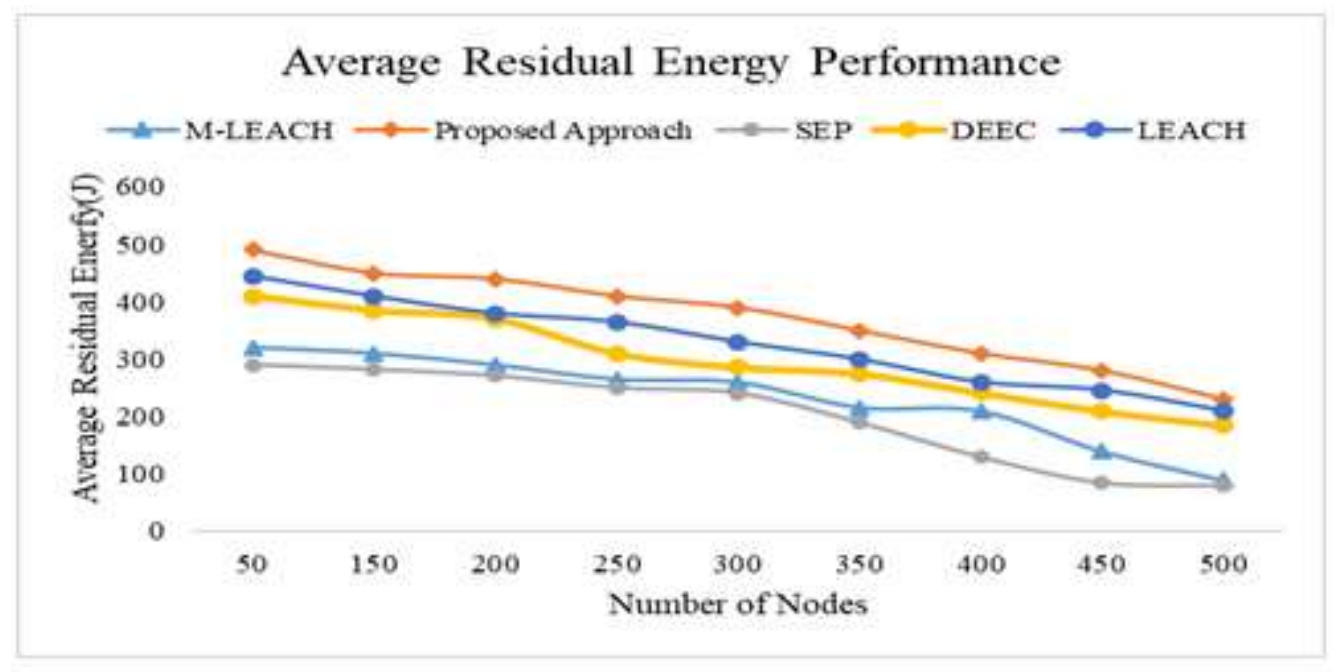

Fig. 16 Residual energy performance 
The complete experimental study shows that the proposed approach achieves better performance in terms of network lifetime, average throughput, energy consumption and average residual energy. The performance obtained using proposed approach shows improved results when compare to existing protocols as discussed before. The average performance is obtained as 202.11, 297, 233.4, 327.3, and 372.2 using SEP, DEEC, M-LEACH, LEACH and Proposed approach.

\section{CONCLUSION}

Coal mine monitoring is considered a challenging task due to hazardous working conditions faced by the underground mines. During last two decades, several techniques have been presented which are mainly focused on cable based monitoring system and sensor network based systems. Sensor network based system shows significant impact on the monitoring due to their efficient data collection and self-configurable nature architecture. However, efficient data collection and transmission to the base station is an important task in this field where existing techniques suffer from data loss resulting in poor monitoring of underground coal mines. In order to overcome this issue, we present Fuzzy Logic based decision making process for packet routing for multi-layer coal mining architecture. According to the proposed approach, energy and distance parameters are used for Cluster head formation. Once the cluster head is selected, Fuzzy Rules are generated by considering energy, expected energy consumption, distance from sink node and residual energy parameters. Based on these fuzzy rules, next hop is selected for routing to develop the energy efficient and better packet routing scheme. Performance of proposed approach is compared with the existing techniques; the comparative study shows that the proposed approach achieves better performance.

\section{REFERENCES}

[1] https://nma.org/category/statistics/.

[2] Joy, G.J. Evaluation of the approach to respirable quartz exposure control in us coal mines. J. Occup. Environ. Hyg. 2012, 9, 65-68.

[3] Trevits, M.; Yuan, L.; Smith, A.; Thimons, E.; Goodman, G. The status of mine fire research in the United States. In Proceedings of the 21st World Mining Congress; Taylor \& Francis Group: Krakow, Poland; London, UK, 2008; pp. 303-308.

[4] Annual Report, 2011 of Chief Inspector of Mines, Punjab. Available online:http://cim.pitb.gov.pk/system/files/CIM_AnnualReport_2011.pdf

[5] Osunmakinde, I.O. Towards safety from toxic gases in underground mines using wireless sensor networks and ambient intelligence. Int. J. Distributed. Sens. Network. 2013.http://dx.doi.org/10.1155/2013/159273

[6] Ni L.M., Liu Y., Zhu Y. China's National Research Project on Wireless Sensor Networks. IEEE Wireless. Communication. 2011; 14:78-83.http://dx.doi.org/10.1109/MWC.2007.4407230

[7] Molina, S., Soto, I., \& Carrasco, R. (2011, 14-16 March). Detection of gases and collapses in underground mines using WSN. Paper presented at the IEEE International Conference on Industrial Technology (ICIT).

[8] http://dx.doi.org/10.1109/ICIT.2011.5754376

[9] Zhao G (2011) Wireless sensor networks for industrial process monitoring and control: a survey. Network Protocols Algorithms 3(1):46-63

[10] http://dx.doi.org/10.5296/npa.v3i1.580

[11] Jiang Q, Ma J, Yang C, Ma X, Shen J, Chaudhry SA (2017a) Efficient end-to-end authentication protocol for wearable health monitoring systems. Computers \&Electrical Engineering. http://dx.doi.org/10.1016/j.compeleceng.2017.03.016.

[12] Zhao, C., Liu, F. and Hai, X., 2013. An application of wireless sensor networks in underground coal mine. International Journal of Future Generation Communication and Networking, 6(5), pp.117-126.http://dx.doi.org/10.14257/ijfgen.2013.6.5.11

[13] Heinzelman, W. B., Chandrakasan, A. P., \&Balakrishnan, H. (October 2002). An applicationspecific protocol architecture for wireless microsensor networks. IEEE Transactions on Wireless Communications, 660-670. 
[14] Lindsey, S., Raghavendra, C., \& Sivalingam, K. M. (September 2002). Data gathering algorithms in sensor networks using energy metrics. IEEE Transactions on Parallel and Distributed Systems, 924-935.http://dx.doi.org/10.1109/TPDS.2002.1036066

[15] Younis, O., \&Fahmy, S. (October 2004). HEED: A hybrid, energy-efficient, distributed clustering approach for ad hoc sensor network. IEEE Transaction on Mobile, Computing, 660 669.http://dx.doi.org/10.1109/TMC.2004.41

[16] Xia, X., Chen, Z., Li, D. and Li, W., 2014. Proposal for efficient routing protocol for wireless sensor network in coal mine Goaf. Wireless personal communications, 77(3), pp.1699-1711. http://dx.doi.org/10.1007/s11277-014-1603-8

[17] Hu, Q., Wu, L., Geng, F. and Cao, C., 2014. A data transmission algorithm based on dynamic grid division for coal Goaf temperature monitoring. Mathematical Problems in Engineering, 2014. http://dx.doi.org/10.1155/2014/652621

[18] Moridi, M.A., Kawamura, Y., Sharifzadeh, M., Chanda, E.K., Wagner, M., Jang, H. and Okawa, H., 2015. Development of underground mine monitoring and communication system integrated ZigBee and GIS. International Journal of Mining Science and Technology, 25(5), pp.811-818.

[19] Kumari, S. and Om, H., 2016. Authentication protocol for wireless sensor networks applications like safety monitoring in coal mines. Computer Networks, 104, pp.137-154. http://dx.doi.org/10.1016/j.comnet.2016.05.007

[20] Ren, P. and Qian, J., 2016. A power-efficient clustering protocol for coal mine face monitoring with wireless sensor networks under channel fading conditions. Sensors, 16(6), p.835.https://doi.org/10.3390/s16060835.

[21] Barnwal, R.P., Bharti, S., Misra, S. and Obaidat, M.S., 2017. UCGNet: wireless sensor network-based active aquifer contamination monitoring and control system for underground coal gasification. International Journal of Communication Systems, 30(1), p. e2852.https://doi.org/10.1002/dac.2852.

[22] Alfonso, I., Goméz, C., Garcés, K. and Chavarriaga, J. Lifetime optimization of Wireless Sensor Networks for gas monitoring in underground coal mining. In 2018, 7th International Conference on Computers Communications and Control (ICCCC) (pp. 224-230). IEEE.https://doi.org/10.1109/ICCCC.2018.8390462. 